\title{
ON THE LOCAL EXISTENCE OF SOLUTIONS OF CERTAIN FUNCTIONAL-DIFFERENTIAL EQUATIONS
}

\author{
ROBERT J. OBERG
}

A natural way of generalizing ordinary differential equations is to permit the unknown function to appear with different values of the argument. "Differential equations with deviating argument" or "functional-differential equations" such as

$$
x^{\prime}(t)=F(t, x(t), x(g(t)))
$$

have been extensively studied, usually under the assumption that $g(t) \leqq t$. The theory for this retarded case is quite satisfactory. See [3] for a good introduction to this subject.

When we make no assumption on $g$, except for continuity, the situation is not nearly so well understood, even for a very simple equation (cf. [4]) like

$$
x^{\prime}(t)=a x(g(t)) \quad(a \text { is a constant }) .
$$

The first question one raises is that of local existence of solutions. Notice that this question is meaningful only around a fixed point of $g$. For if $g\left(t_{0}\right) \neq t_{0}$ we may specify $x(t)$ arbitrarily in a neighborhood of $t_{0}$ and determine $x(t)$ in a neighborhood of $g\left(t_{0}\right)$ by the differential equation. We will show in particular that if $g$ is continuous then (2) has a solution in the neighborhood of a fixed point for every value $a$. Our results generalize those of Anderson [1], who was able to conclude in general only that (2) has a solution for certain $g$ or for certain values of $a$.

More generally, we consider the functional-differential equation

$$
x^{\prime}(t)=F(t, x(t), x(g(t, x(t))))
$$

where the argument depends not only on the independent variable but also on the dependent variable, a type of equation considered by Driver [2] in the retarded case. A special case of (3) is $x^{\prime}(t)=a x(g(x(t)))$, the equation involving "nesting" suggested by Anderson. We wish to find a solution of (3), defined in a neighborhood of $t_{0}$, that satisfies the initial condition $x\left(t_{0}\right)=x_{0}$. Again local existence is nontrivial only around a "fixed point"-i.e. when $g\left(t_{0}, x_{0}\right)=t_{0}$. Actually our local existence theorem will apply to systems; we assume that the functions $x$ and $F$ take their values in Euclidean $n$-space.

Received by the editors February 8, 1968. 
We first prove a local existence theorem for (3) under the assumption that $F$ and $g$ are Lipschitz continuous. An easy corollary of the proof of this theorem will be the uniqueness of the solution of the initial value problem in the special case that the fixed point is "contracting." We show by example that in general-even for $F$ Lipschitz and in the neighborhood of a fixed point of $g$-uniqueness breaks down. Finally, for the simpler equation (1), we prove a "Peano" type existence theorem that requires only the assumption that $g$ and $F$ are continuous.

Notation. For vectors $x=\left(x_{1}, \cdots, x_{n}\right)$ in Euclidean $n$-space $R^{n}$ we introduce the length $|x|=\max \left|x_{i}\right|$. Let $C(R)$ denote the space of bounded continuous functions $x: R \rightarrow R^{n}$ with norm $\|x\|_{\infty}=$ $\sup \{|x(t)|: t \in R\}$. Let $C^{1}(R)$ denote the space of bounded continuously differentiable functions $x: R \rightarrow R^{n}$ whose derivatives are bounded, with norm $\|x\|=\|x\|_{\infty}+\left\|x^{\prime}\right\|_{\infty} . C^{1}(R)$ is a complete metric space with metric $d(x, y)=\|x-y\|, F(t, x, y)$ is a function from $R^{2 n+1}$ into $R^{n}$.

THEOREM 1. Suppose that $F(t, x, y)$ is continuous and satisfies a uniform Lipschitz condition in the closed domain $\left|t-t_{0}\right| \leqq a,\left|x-x_{0}\right| \leqq b$, $\left|y-x_{0}\right| \leqq b$. Suppose that the function $g(t, x)$ is continuous on $\left|t-t_{0}\right|$ $\leqq a,\left|x-x_{0}\right| \leqq b$ and satisfies a uniform Lipschitz condition there. Assume $g\left(t_{0}, x_{0}\right)=x_{0}$. Then there exists $h>0$ such that on the interval $\left|t-t_{0}\right| \leqq h(3)$ has a solution satisfying $x\left(t_{0}\right)=x_{0}$.

REMARK. We say that $x(t)$ is a solution of (3) on an interval $I$ if $x(t)$ is defined for $t \in I \cup_{o}(I, x(l))$ and satisfies (3) for $t \in I$.

Proof. We will apply the contraction mapping theorem to find a local solution of the equivalent integral equation

$$
x(t)=x_{0}+\int_{t_{0}}^{t} F(t, x(s), x(g(s, x(s)))) d s .
$$

Let $L$ be the Lipschitz constant for $F$, so that the Lipschitz condition reads $|F(t, x, y)-F(t, \tilde{x}, \tilde{y})| \leqq L \max (|x-\tilde{x}|,|y-\tilde{y}|)$. Let $K$ be the Lipschitz constant for $g$. Let $M$ be the supremum of $|F(t, x, y)|$ taken over $\left|t-t_{0}\right| \leqq a,\left|x-x_{0}\right| \leqq b,\left|y-x_{0}\right| \leqq b$. Choose $\delta>0$ by the continuity of $g$ so that

$$
\left|t-t_{0}\right| \leqq \delta \quad \text { implies }\left|g\left(t, x_{0}\right)-g\left(t_{0}, x_{0}\right)\right| \leqq 1 / 24 L .
$$

Then define $h$ by

$$
3 h=\min (a, b / M, 1 / 2 L(1+3 M K), 1 / 72 M L K, 1 / 6 L, \delta) .
$$

Let $S$ be the space of functions $x \in C^{1}(R)$ satisfying $x\left(t_{0}\right)=x_{0},\left\|x-x_{0}\right\|_{\infty}$ 
$\leqq b,\left\|x^{\prime}\right\|_{\infty} \leqq 3 M$. Then $S$ is a complete metric space, since it is a closed subspace of $C^{1}(R)$.

Now take $j: R \rightarrow R$ to be any continuously differentiable function which satisfies:

(i) $j(t)=1\left(\left|t-t_{0}\right| \leqq h\right)$,

(ii) $j(t)=0\left(\left|t-t_{0}\right| \geqq 3 h\right)$,

(iii) $0 \leqq j(t) \leqq 1$ (all $t \in R$ ),

(iv) $\left|\left(t-t_{0}\right) j^{\prime}(t)\right| \leqq 2$ (all $t \in R$ ).

(The last condition can be assured by making $\left|j^{\prime}(t)\right| \leqq 2 / 3 h$.)

Define an operator $U$ on $S$ by

$$
\begin{aligned}
U x(t) & =x_{0}+j(t) \int_{t_{0}}^{t} F(s, x(s), x(g(s, x(s)))) d s \quad \text { if }\left|t-t_{0}\right| \leqq 3 h, \\
& =x_{0} \quad \text { otherwise. }
\end{aligned}
$$

Notice that $U x(t)-U y(t)=0=(U x)^{\prime}(t)-(U y)^{\prime}(t)$ for $\left|t-t_{0}\right| \geqq 3 h$, so in making estimates for these differences we may assume $\left|t-t_{0}\right| \leqq 3 h$.

We first check that $U$ maps $S$ into itself. Let $x \in S$. Then clearly $U x$ is continuously differentiable and $U x\left(t_{0}\right)=x_{0}$. Also $\left|U x(t)-x_{0}\right|$ $\leqq\left|t-t_{0}\right| \cdot M \leqq 3 h M \leqq b$ if $\left|t-t_{0}\right| \leqq 3 h$. Thus $\left\|U x-x_{0}\right\|_{\infty} \leqq b$. Finally $\left|(U x)^{\prime}(t)\right| \leqq\left|j^{\prime}(t)\left(t-t_{0}\right) M\right|+M \leqq 3 M$ (by properties (iii) and (iv) of $j(t))$, and so $\left\|(U x)^{\prime}\right\|_{\infty} \leqq 3 M$. Thus $U x \in S$.

Next we verify that $U$ is a contraction operator. Let $x, y \in S$. Then, for $\left|t-t_{0}\right| \leqq 3 h$, we have, using the Lipschitz condition for $F$,

$$
\begin{aligned}
& |U x(t)-U y(t)| \\
& \leqq\left|t-t_{0}\right| \cdot L \cdot \sup \max (|x(s)-y(s)|,|x(g(s, x(s)))-y(g(s, y(s)))|)
\end{aligned}
$$

where $s$ ranges over the set $\left\{s:\left|s-t_{0}\right| \leqq\left|t-t_{0}\right|\right\}$. Now

$$
\begin{aligned}
& |x(g(s, x(s)))-y(g(s, y(s)))| \\
& \quad \leqq|x(g(s, x(s)))-y(g(s, x(s)))|+|y(g(s, x(s)))-y(g(s, y(s)))| \\
& \quad \leqq\|x-y\|_{\infty}+|g(s, x(s))-g(s, y(s))| \cdot 3 M
\end{aligned}
$$

(applying the mean value theorem to each component of $y$ and using inequality $\left\|y^{\prime}\right\|_{\infty} \leqq 3 M$ )

$$
\leqq\|x-y\|_{\infty}+3 M K\|x-y\|_{\infty}
$$

(using Lipschitz condition for $g$ ). We thus have $\left(\left|t-t_{0}\right| \leqq 3 h\right.$ )

$$
\begin{aligned}
|U x(t)-U y(t)| & \leqq\left|t-t_{0}\right| \cdot L \cdot(1+3 M K)\|x-y\|_{\infty} \\
& \leqq 3 h \cdot L \cdot(1+3 M K)\|x-y\|_{\infty} \leqq \frac{1}{2}\|x-y\|_{\infty}
\end{aligned}
$$

by (6). Hence 


$$
\|U x-U y\|_{\infty} \leqq \frac{1}{2}\|x-y\|_{\infty} .
$$

Now we estimate $\left|(U x)^{\prime}(t)-(U y)^{\prime}(t)\right|$ for $\left|t-t_{0}\right| \leqq 3 h$. We have $\left|(U x)^{\prime}(t)-(U y)^{\prime}(t)\right|$

$$
\begin{aligned}
\leqq & \left|j^{\prime}(t) \int_{t_{0}}^{t}[F(s, x(s), x(g(s, x(s))))-F(s, y(s), y(g(s, y(s))))] d s\right| \\
& +|j(t)[F(t, x(t), x(g(t, x(t))))-F(t, y(t), y(g(t, y(t))))]| .
\end{aligned}
$$

Using properties (iii) and (iv) of $j(t)$ we find (where the sup is again taken over the set $\left.\left\{s:\left|s-t_{0}\right| \leqq\left|t-t_{0}\right|\right\}\right)$

$\left|(U x)^{\prime}(t)-(U y)^{\prime}(t)\right|$

$$
\begin{aligned}
& \leqq 3 \sup _{s}|F(s, x(s), x(g(s, x(s))))-F(s, y(s), y(g(s, y(s))))| \\
& \leqq 3 L \sup _{s} \max (|x(s)-y(s)|,|x(g(s, x(s)))-y(g(s, y(s)))|)
\end{aligned}
$$

(by the Lipschitz condition for $F$ ). Now

$$
\begin{aligned}
|x(s)-y(s)| & =\left|\int_{t_{0}}^{0}\left(x^{\prime}(r)-y^{\prime}(r)\right) d r\right| \quad\left(\text { since } x\left(t_{0}\right)=x_{0}=y\left(t_{0}\right)\right) \\
& \leqq 3 h\left\|x^{\prime}-y^{\prime}\right\|_{\infty} \quad\left(\text { since }\left|t-t_{0}\right| \leqq 3 h\right) \\
& \leqq \frac{1}{6 L}\left\|x^{\prime}-y^{\prime}\right\|_{\infty} \quad \text { by }(6) .
\end{aligned}
$$

Also

$$
\begin{aligned}
|x(g(s, x(s)))-y(g(s, y(s)))| & \leqq|x(g(s, x(s)))-y(g(s, x(s)))| \\
& +|y(g(s, x(s)))-y(g(s, y(s)))| .
\end{aligned}
$$

The first term is

$$
\begin{aligned}
\left|\int_{o\left(t_{0}, x_{0}\right)}^{o(s, x(s))}\left(x^{\prime}(r)-y^{\prime}(r)\right) d r\right| \\
\quad \leqq\left\|x^{\prime}-y^{\prime}\right\|_{\infty}\left(\left|g(s, x(s))-g\left(t_{0}, x_{0}\right)\right|\right) \\
\quad \leqq\left\|x^{\prime}-y^{\prime}\right\|_{\infty}\left(\left|g(s, x(s))-g\left(s, x_{0}\right)\right|+\left|g\left(s, x_{0}\right)-g\left(t_{0}, x_{0}\right)\right|\right) \\
\quad \leqq\left\|x^{\prime}-y^{\prime}\right\|_{\infty}\left[K\left|x(s)-x_{0}\right|+\frac{1}{24 L}\right]
\end{aligned}
$$

(by Lipschitz condition for $g$ and (5), since $\left|s-t_{0}\right| \leqq 3 h \leqq \delta$ )

$$
\leqq\left\|x^{\prime}-y^{\prime}\right\|_{\infty}\left[K \cdot 3 M \cdot 3 h+\frac{1}{24 L}\right]
$$


(applying the mean value theorem to each component of $x$ and using the inequalities $\left.\left\|x^{\prime}\right\|_{\infty} \leqq 3 M,\left|s-t_{0}\right| \leqq 3 h\right)$

$$
\leqq(1 / 12 L)\left\|x^{\prime}-y^{\prime}\right\|_{\infty} \quad \text { by }(6) \text {. }
$$

Again, applying the mean value theorem to $y$ and using the Lipschitz condition for $g$, the second term in (11) is

$$
\begin{aligned}
& \leqq 3 M|g(s, x(s))-g(s, y(s))| \leqq 3 M K|x(s)-y(s)| \\
& =3 M K\left|\int_{t_{0}}^{\bullet}\left[x^{\prime}(r)-y^{\prime}(r)\right] d r\right| \leqq 3 M K \cdot 3 h \cdot\left\|x^{\prime}-y^{\prime}\right\|_{\infty} \\
& \leqq \frac{1}{24 L}\left\|x^{\prime}-y^{\prime}\right\|_{\infty} \leqq \frac{1}{12 L}\left\|x^{\prime}-y^{\prime}\right\|_{\infty}
\end{aligned}
$$

by (6). Combining our estimates for the two terms in (11), we find that

$$
|x(g(s, x(s)))-y(g(s, y(s)))| \leqq(1 / 6 L)\left\|x^{\prime}-y^{\prime}\right\|_{\infty} .
$$

It now follows from (9), (10), and (12) that $\left|(U x)^{\prime}(t)-(U y)^{\prime}(t)\right|$ $\leqq 3 L \cdot(1 / 6 L)\left\|x^{\prime}-y^{\prime}\right\|_{\infty}=\frac{1}{2}\left\|x^{\prime}-y^{\prime}\right\|_{\infty}$. Hence

$$
\left\|(U x)^{\prime}-(U y)^{\prime}\right\|_{\infty} \leqq \frac{1}{2}\left\|x^{\prime}-y^{\prime}\right\|_{\infty} \text {. }
$$

Combining (8) and (13) we find that $\|U x-U y\| \leqq \frac{1}{2}\|x-y\|$ and so $U$ is a contraction operator.

Therefore we can conclude from the contraction mapping theorem that $U$ has a unique fixed point $x(t)$ in $S$. On $\left|t-t_{0}\right| \leqq h$ (the interval where $j(t) \equiv 1) x(t)$ satisfies (4). This completes the proof of the theorem.

The fixed point $x(t)$ is not necessarily a unique solution of (4), because of its dependence on the choice of $j(t)$. We can try to eliminate this dependence by introducing the space $S_{0}$ of restrictions to $\left|t-t_{0}\right| \leqq h$ of functions in $S$. Then if

$$
x(t) \in S_{0} \quad \text { implies } x(g(t, x(t))) \in S_{0}
$$

it follows that the operator $U$ defined in (7) is just $U x(t)$ $=x_{0}+\int_{t_{0}}^{t} F(s, x(s), x(g(s, x(s)))) d s\left(\left|t-t_{0}\right| \leqq h\right) . U$ maps $S_{0}$ into itself and does not depend on $j(t)$. Accordingly the local solution of (4) is unique if (14) is satisfied.

Definition. The function $g(t, x)$ is said to be contracting at $\left(t_{0}, x_{0}\right)$ if for $(t, x)$ close to $\left(t_{0}, x_{0}\right),\left|g(t, x)-t_{0}\right| \leqq\left|t-t_{0}\right|$.

It is evident that (14) is satisfied if $g$ is contracting at $\left(t_{0}, x_{0}\right)$. We thus have 
Theorem 2. Let $F(t, x, y)$ and $g(t, x)$ satisfy the hypotheses of Theorem 1. Suppose also that $g(t, x)$ is contracting at $\left(t_{0}, x_{0}\right)$. Then the local solution of (3) satisfying $x\left(t_{0}\right)=x_{0}$ is unique.

If $g$ is not contracting at a fixed point local uniqueness of the solution of the initial value problem may well fail. For example consider the following initial value problem:

$$
x^{\prime}(t)=x(2 t), \quad x(0)=1 .
$$

There are infinitely many $C^{\infty}$ solutions of (15) defined for $t \geqq 0$. For we may let $\phi(t)$ be an arbitrary function that is $C^{\infty}$ on $[1,2]$ and flat at 1 and 2 . We then define $x(t)$ on $[1 / 2,1]$ by $x(t)=c+\int_{1 / 2}^{t} \phi(2 s) d s$, where $c$ is chosen to make $x(1)=\phi(1)$. We may then inductively define $x(t)$ on $\left[1 / 2^{n}, 1 / 2^{n-1}\right]$ in a similar manner. It is not difficult to check that $\lim _{t \rightarrow 0+} x(t)$ exists. Then $x(t)$ is a $C^{\infty}$ solution of $x^{\prime}(t)=x(2 t)$ $(t \geqq 0)$. Multiplying by a suitable constant we can make $x(0)=1$. It follows that the initial value problem (15) has infinitely many solutions, since there was so much latitude in our choice of $\phi(t)$.

This last example also helps to explain the difficulty of the proof of local existence in general. We try to construct a space $S$ of functions defined on a small interval $\left[t_{0}-h, t_{0}+h\right]$. But in general $g$ will not map this interval into itself, and so the linear operator we define will not map the space $S$ into itself. We overcame this difficulty by considering functions defined on the whole real line and modifying the integral by a suitable function $j(t)$. In the case $g=g(t)$ there is another artifice we can use: modify $g$ slightly so that it maps a small interval into itself but coincides with the original $g$ in a very small neighborhood of $t_{0}$. Using the Schauder fixed point theorem, we are able to show that (1) possesses a local solution assuming only the continuity of $g$ and $F$. (As before $x$ and $F$ are $n$-vectors.)

THEOREM 3. Suppose that $g\left(t_{0}\right)=t_{0}$ and $g(t)$ is continuous over $\left|t-t_{0}\right| \leqq a$. Suppose that $F(t, x, y)$ is continuous on $\left|t-t_{0}\right| \leqq a,\left|x-x_{0}\right|$ $\leqq b,\left|y-x_{0}\right| \leqq b$. Then there exists $h>0$ such that on the interval $\left|t-t_{0}\right|$ $\leqq h$ (1) has a solution satisfying $x\left(t_{0}\right)=x_{0}$.

Proof. We will apply the Schauder fixed point theorem to find a local solution of the equivalent integral equation

$$
x(t)=x_{0}+\int_{t_{0}}^{t} F(s, x(s), x(g(s))) d s .
$$

Let $M$ be the supremum of $|F(t, x, y)|$ taken over $\left|t-t_{0}\right| \leqq a$, $\left|x-x_{0}\right| \leqq b,\left|y-x_{0}\right| \leqq b$. Set 


$$
h_{1}=\min (a, b / M) .
$$

Let $I_{1}=\left[t_{0}-h_{1}, t_{0}+h_{1}\right]$. By the continuity of $g$ pick $h, 0<h \leqq h_{1}$, so that $g(I) \subset I_{1}$, where $I=\left[t_{0}-h, t_{0}+h\right]$. Define $g_{1}: I_{1} \rightarrow I_{1}$ by

$$
\begin{aligned}
g_{1}(t) & =g\left(t_{0}-h\right) & & t_{0}-h_{1} \leqq t \leqq t_{0}-h, \\
& =g(t) & & t_{0}-h \leqq t \leqq t_{0}+h, \\
& =g\left(t_{0}+h\right) & & t_{0}+h \leqq t \leqq t_{0}+h_{1} .
\end{aligned}
$$

Then $g_{1}$ is continuous and $g_{1}\left(I_{1}\right) \subset I_{1}$.

Let $X$ be the Banach space of continuous functions $x: I_{1} \rightarrow R^{n}$ with sup norm. Let $S$ be the subset of $X$ consisting of the functions $x$ that satisfy $\left\|x-x_{0}\right\| \leqq b$. Then $S$ is clearly a closed convex subset of $X$. Define the operator $U$ by

$$
U x(t)=x_{0}+\int_{t_{0}}^{t} F\left(s, x(s), x\left(g_{1}(s)\right)\right) d s .
$$

Then $U$ maps $S$ into itself. For let $x \in S$. Then $U x: I_{1} \rightarrow R^{n}$ is continuous, and, for $\left|t-t_{0}\right| \leqq h_{1},\left|U x(t)-x_{0}\right| \leqq h_{1} \cdot M \leqq b$ by (17). The mapping $U$ is clearly continuous, since the function $F(t, x, y)$ is uniformly continuous on the closed set $\left|t-t_{0}\right| \leqq h_{1},\left|x-x_{0}\right| \leqq b,\left|y-x_{0}\right| \leqq b$.

Finally we check that the image $U(S)$ is compact. Indeed, the family of functions in $U(S)$ is uniformly bounded (by $\left|x_{0}\right|+b$ ) and equicontinuous $\left(y \in U(S)\right.$ implies $y=U x$ with $x \in S$, and so $\left|y\left(t_{1}\right)-y\left(t_{2}\right)\right|$ $\left.\leqq\left|\int_{t_{2}}^{t_{1}} F\left(s, x(s), x\left(g_{1}(s)\right)\right) d s\right| \leqq M\left|t_{1}-t_{2}\right|\right)$. Hence $U(S)$ is compact by the Arzela-Ascoli Theorem.

It then follows from the Schauder-Leray fixed point theorem that $U$ has a fixed point $x(t)$ defined on $I_{1}$. Restricting to $\left|t-t_{0}\right| \leqq h$ we see that $x(t)$ is a local solution of (16).

The author wishes to thank the referee for calling his attention to several additional references. In $1921 \mathrm{~W}$. B. Fite [6] considered essentially equation (1). He proved an existence and uniqueness theorem for $g(t)$ subject to the condition $\left(^{*}\right)\left|g(t)-t_{0}\right| \leqq\left|t-t_{0}\right|$. Since then numerous rediscoveries and variations of this result have been published ([5], [1], [7], and others). The present paper is different in two respects. First a local existence theorem is established without requiring any assumption such as $\left(^{*}\right)$, although then uniqueness is lost. (An interesting question is how to get a well-posed problem for an equation like $x^{\prime}(t)=x(2 t)$ in an interval containing 0 .) Second, the function $g$ is permitted to depend on $x(t)$. A paper by V. P. Skripnik [8] also considers equations with $g$ depending on $x(t)$. Conditions are obtained for a solution to exist on an interval $[a, b]$. However, it appears that a local existence theorem cannot be deduced from this result without 
making some additional assumption on $F$ and $g$. An interesting feature of this paper is the "multipoint" boundary value problem that is solved: a solution $x(t)$ is found whose components $x_{i}(t)$ satisfy initial conditions $x_{i}\left(t_{i}\right)=x_{i 0}$ at different points $t_{i}$.

\section{REFERENCES}

1. D. R. Anderson, An existence theorem for a solution of $f^{\prime}(x)=F(x, f(g(x)))$, SIAM Rev. 8 (1966), 359-362.

2. R. Driver, Existence theory for a delay-differential system, Contributions to Differential Equations 1 (1963), 317-336.

3. L. El'sgol'ts, Introduction to the theory of differential equations with deviating argument, Holden-Day, San Francisco, Calif., 1966.

4. W. R. Utz, The equation $f^{\prime}(x)=a f(g(x))$, Bull. Amer. Math. Soc. 71 (1965), 138.

5. S. Doss and S. K. Nasr, On the functional equation $d y / d x=f(x, y(x), y(x+h))$, $h>0$, Amer. J. Math. 75 (1953), 713-716.

6. W. B. Fite, Properties of the solutions of certain functional differential equations, Trans. Amer. Math. Soc. 22 (1921), 311-319.

7. V. P. Skripnik, Systems with transformed argument. Boundaryvalue problems and the Cauchy problem, Mat. Sb. (N.S.) 62 (1963), 385-396.

8. - Systems with transformed argument in the case where the transformed argument depends on its solutions, Mat. Sb. (N.S.) 68 (1965), 274-281.

HARVARD UNIVERSITY 